\title{
Preface Issue 1-2013
}

\section{Hans-Christoph Grunau}

(C) Deutsche Mathematiker-Vereinigung and Springer-Verlag Berlin Heidelberg 2013

Annette Werner provides an introduction to the research area of "Non-Archimedean analytic spaces", i.e. analytic geometry over complete non-Archimedean fields. These fields arise in algebra and number theory and the corresponding geometry looks completely different from the usual analytic, projective or differential geometry. The author presents a new approach to this kind of arithmetic geometry with "better" topological properties.

Hans Grauert passed away in September 2011. Alan Huckleberry has written a very illustrative and informative obituary in which he reviews some of the most influential and fundamental works of "one of the greatest giants of the second half of the 20th century" in complex geometry. We learn about the-often quite classicalcomplex analytic background, the key achievements, the main implications and some of the really modern ideas of these works. While the precise formulation of the mathematics requires some abstract and deep concepts, the present article focusses on the underlying analytical intuition and geometrical imagination. Moreover, Hans Grauert's interactions with his advisors, friends, coworkers, mathematical companions and students are outlined in some detail.

Recently released books on nonlinear potential theory on metric spaces, on statistical regression for specific kinds of data, and on random matrices in mathematical physics are extensively discussed and reviewed.

Every four years the (managing) editor of the Jahresbericht is elected by the members of the DMV. This is the beginning of my second term of office, which will last until the end of 2016. It is my hope that all the changes, the Jahresbericht had to undergo during the past four years, will help it to become internationally more visible as

\footnotetext{
H.-Ch. Grunau ( $\bowtie)$

Institut für Analysis und Numerik, Fakultät für Mathematik, Otto-von-Guericke-Universität, Postfach 4120, 39016 Magdeburg, Germany

e-mail: hans-christoph.grunau@ovgu.de
} 
a journal devoted to accessible articles on important and interesting issues of mathematics and to reviews of recent books of general interest. Also the editorial board changes every four years. I would like to take the opportunity to thank the members of the previous board for the very enjoyable and fruitful cooperation. The new editorial board consists of Felix Finster, Hansjörg Geiges, Martin Grothaus, Martin Hanke, Michael Hinze, Gabriele Nebe, Guido Schneider, Wolfgang Soergel, Thomas Bartsch, who is responsible for the book reviews, and myself. I am confident that we all together will collect a number of interesting contributions from many different vibrant areas of mathematics. 